OPEN ACCESS

Edited by: Francesco Cellini, Fondazione Policlinico A. Gemelli IRCCS, Italy

Reviewed by: Valentina Lancellotta, Catholic University of the Sacred Heart, Italy Ramin Sadeghi, Mashhad University of Medical Sciences, Iran

*Correspondence: Clelia Di Carlo clelia.dicarlo@ospedaliriuniti.marche.it

Specialty section: This article was submitted to Radiation Oncology, a section of the journal Frontiers in Oncology

Received: 18 January 2021 Accepted: 14 June 2021 Published: 01 July 2021

Citation:

Di Carlo C, di Benedetto M, Vicenzi L, Costantini S, Cucciarelli F. Fenu F, Arena E, Mariucci C, Montisci M, Panni V, Patani F, Valenti M, Palucci A, Burroni $L$ and Mantello G (2021) FDG-PET/CT in the

Radiotherapy Treatment Planning of Locally Advanced Anal Cancer: A Monoinstitutional Experience. Front. Oncol. 11:655322. doi: 10.3389/fonc.2021.655322

\section{FDG-PET/CT in the Radiotherapy Treatment Planning of Locally Advanced Anal Cancer: A Monoinstitutional Experience}

\author{
Clelia Di Carlo ${ }^{1 *}$, Maika di Benedetto ${ }^{1}$, Lisa Vicenzi ${ }^{1}$, Sara Costantini ${ }^{1}$, \\ Francesca Cucciarelli ${ }^{1}$, Francesco Fenu ${ }^{1}$, Eleonora Arena ${ }^{1}$, Cristina Mariucci ${ }^{1}$, \\ Maria Montisci ${ }^{1}$, Valeria Panni ${ }^{1}$, Fabiola Patani ${ }^{1}$, Marco Valenti ${ }^{2}$, Andrea Palucci ${ }^{3}$, \\ Luca Burroni $^{3}$ and Giovanna Mantello ${ }^{1}$ \\ ${ }^{1}$ Department of Radiation Oncology, Ospedali Riuniti Umberto I', GM Lancisi, G Salesi, Ancona, Italy, ${ }^{2}$ Department of \\ Medical Physics, Ospedali Riuniti Umberto $1^{\circ}$. GM Lancisi, G Salesi, Ancona, Italy, ${ }^{3}$ Department of Nuclear Medicine, \\ Ospedali Riuniti Umberto I’, GM Lancisi, G Salesi, Ancona, Italy
}

Aims: Radiotherapy with concurrent 5-fluorouracil/mitomycin-C based chemotherapy has been established as definitive standard therapy approach for anal cancer. Intensity Modulated Radiotherapy (IMRT) leads to a precise treatment of the tumor, allowing dose escalation on Gross Tumor Volume (GTV), with a surrounding healthy tissues sparing. Our study assessed the impact of 18-Fluorodeoxyglucose positron emission tomography (18FDG-PET/CT) on the radiotherapy contouring process and its contribution to lymphatic spread detection, resulting to a personalization of Clinical Target Volume (CTV) and dose prescription.

Methods: Thirty-seven patients, with histologically proven squamous cell carcinoma of the anal canal (SCCAC) were analyzed. All patients were evaluated with history and physical examination, trans-anal endoscopic ultrasound, pelvis magnetic resonance imaging (MRI), computed tomography (CT) scans of the chest, abdomen and pelvis and planning 18FDG-PET/CT. The GTV and CTV were drawn on CT, MRI and 18FDGPET/CT fused images.

Results: Thirty-four (91\%) out of 37 patients presented lymph nodes involvement, in one or more areas, detected on 18FDG-PET/CT and/or MRI. The 18FDG-PET/CT showed positive lymph nodes not detected on MRI imaging (PET+, MRI-) in 14/37 patients (38\%). In 14 cases, 18FDG-PET/CT allowed to a dose escalation in the involved nodes. The 18FDG-PET/CT fused images led to change the stage in 5/37(14\%) cases: four cases from NO to N1 (inguinal lymph nodes) and in one case from M0 to M1 (common iliac lymph nodes).

Conclusions: The 18FDG-PET/CT has a potentially relevant impact in staging and target volume delineation/definition in patients affected by anal cancer. In our experience, clinical stage variation occurred in $14 \%$ of cases. More investigations are needed to define the role of 18FDG-PET/CT in the target volume delineation of anal cancer.

Keywords: anal cancer, 18FDG PET/CT, radiotherapy planning, dose escalation, target volume definition 


\section{INTRODUCTION}

Anal cancer is a rare disease accounting for $1-2 \%$ of digestive tract tumor in Europe and it is strongly related to HPV infection in as many as $90 \%$ of cases.

At diagnosis, $50 \%$ of anal cancer results confined to the primary site, $30 \%$ presents regional lymph nodes (LN) involvement while distant metastases are reelevated in less than $10 \%$ of cases $(1,2)$.

In locally advanced stage, the standard of care is represented by concurrent radio-chemotherapy with 5-fluorouracil and mitomycin C. Surgical resection is an option for nonresponders or recurrent disease (3).

For locoregional staging, magnetic resonance imaging (MRI) represents the gold standard in detecting tumor extension and the involvement of adjacent structures such as muscles and soft tissues. Thorax and abdomen computed tomography (CT) is used to assess distant extent of spread, mainly metastasis in liver and lungs. In recent years, 18-Fluorodeoxyglucose (18FDG) positron emission tomography (PET/CT) is having an increasing role in staging and treatment planning of anal carcinoma, because of the high 18FDG-PET/CT avidity of this tumor (4-6).

In a recent meta-analysis of 17 studies, 18FDG-PET/CT showed a sensitivity of $99 \%$ in the detection of primary tumor compared to $67 \%$ of contrast enhanced CT. Moreover, 18FDGPET/CT had an overall sensitivity of $93 \%$ and specificity of $76 \%$ for inguinal LN identification (7).

Currently, 18FDG-PET/CT is not part of routine staging in anal cancer and does not replace diagnostic CT. Nevertheless, several studies have reported the usefulness of pre-treatment 18FDG-PET/CT to better identify the extension of disease as well as to define the clinical volumes for radiation therapy (1-8).

The aim of the present study is to analyze the potential impact of 18FDG-PET/CT in the staging and target volume delineation of patients affected by anal cancer candidate to curative radio-chemotherapy.

\section{METHODS}

We retrospectively analyzed 37 patients with histologically proven squamous cell carcinoma of the anal canal (SCCAC), treated in our Institute between May 2012 and September 2020.

\section{Inclusion Criteria}

From the total of anal cancer patients treated in our Institute, we selected patients with SCCAC that performed, for clinical staging, trans-anal endoscopic ultrasound, pelvis MRI, total body CT scans with contrast enhancement and planning 18FDG/PET-CT. We included patients with stages I-IV disease.

\section{External Beam Radiotherapy}

For radiotherapy treatment, simulation was performed in supine position with a head rest and knee fixation. A planning CT was acquired from the diaphragm to the proximal diaphysis of the femur, with a slice thickness of $2 \mathrm{~mm}$ and adequate bladder filling.

\section{The 18FDG-PET/CT Acquisition}

Within one week from planning CT, all patients underwent planning 18FDG-PET/CT in the Nuclear Medicine Department in treatment position using a GE Discovery 690 PET/CT scanner with a multi-detector-row CT component. Patients were fasted for at least $6 \mathrm{~h}$ prior to scanning. Blood glucose was assessed before starting the diagnostic investigation. The value of $200 \mathrm{mg} /$ $\mathrm{dl}$ was identified as the upper limit of glucose blood level allowed to proceed with the scan. PET/CT images were acquired $60 \pm$ 5 min after an intravenous injection of 18FDG. The dose was administered based on patient's weight $(3.0 \mathrm{MBq} / \mathrm{kg})$. The PET-CT scan was performed in caudal-cranial direction with 3 min acquisition time per bed position from the base of skull to the middle of the thigh. All images were evaluated by two medical experts in nuclear medicine. PET images analysis was conducted based on qualitative and semi-quantitative analysis. Maximal standardized uptake value (SUVmax) was obtained drawing a Volumes of Interest (VOI) on any suspected pathological LN detected.

Moreover, the MRI imaging and the 18FDG-PET/CT were reviewed by an expert radiologist and expert nuclear medicine physician during multidisciplinary discussions.

\section{Radiotherapy Volume Delineation and Planning}

Pelvis MRI, contrast enhanced CT and planning 18FDG-PET/CT were fused with radiotherapy planning CT using automatic coregistration and manually corrected when necessary.

The primary clinical target volume (CTV T) was delineated including gross tumor volume (GTV T), the entire anal canal and sphincter muscles as recommended by contouring atlas guidelines (9-11). Ten millimeters were added to CTV $\mathrm{T}$ to obtain the planning target volume (PTV T).

The nodal CTV (CTV N) included the mesorectum (delineated separately and expanded of $10 \mathrm{~mm}$ to define the internal margin), internal, external, presacral, obturator and inguinal LN areas and ischiorectal fossa. Common iliac LN was included only when involved. Seven millimeters margin were added to CTV $\mathrm{N}$ to generate PTV $\mathrm{N}$ including mesorectal expansion.

The MRI and/or 18FDG-PET/CT positive LN were delineated as GTV $\mathrm{N}$ and expanded of $5 \mathrm{~mm}$ to generate PTV.

\section{External Beam Radiotherapy}

A total dose of $45 \mathrm{~Gy}$ (1.8 Gy/die) was delivered to CTV N with a simultaneous integrated boost (SIB) up to $54 \mathrm{~Gy}(2.16 \mathrm{~Gy} / \mathrm{die}$, $\mathrm{EQD} 2[\alpha / \beta 10]=54.7 \mathrm{~Gy})$ to $\mathrm{CTV} \mathrm{T}$. When indicated, an additional sequential boost on residual disease (GTV T) up to 59 Gy was prescribed. Moreover, on pathological LN (GTV N), we prescribed a SIB with total dose of 50-54 Gy (2-2.16 Gy/die, $\mathrm{EQD} 2[\alpha / \beta 10]=54.7 \mathrm{~Gy})$.

Intensity Modulated Radiotherapy (IMRT) treatment planning was elaborated for all patients. According to our Institute protocol, daily image guided radiotherapy (IGRT) with cone-beam CT (CBCT) was performed before treatment. 


\section{Chemotherapy}

All patients received concomitant chemotherapy with 5fluorouracil $(5 \mathrm{FU}) /$ mitomycin-C as recommended by Italian Association of Radiation Oncology (AIRO) and European guidelines: intravenous continuous infusion of 5-FU 1,000 mg/ $\mathrm{m}^{2} /$ day, days 1-4 and 29-32; Mitomycin $10 \mathrm{mg} / \mathrm{m}^{2}$, bolus days 1 and 29 (1).

\section{RESULTS}

A total of 37 patients with SCCAC were included in the study, 28 (76\%) female and nine (24\%) male. Median age was 55 years (range 40-88). Patients' characteristics and clinical stage are reported in Table $\mathbf{1}$.

The median follow-up was 19 months (range 2-62). At the last follow-up, according to Response Evaluation Criteria In Solid Tumors (RECIST), 33 (89\%) patients had a disease remission while three $(8 \%)$ patients and one $(3 \%)$ patient had local and systemic progression of disease, respectively.

In 34/37 (91\%) patients there was lymph nodal involvement detected on 18FDG-PET/CT and/or MRI. The 18FDG-PET/CT showed positive LN not detected on MRI imaging in 14/37 (38\%) patients: two cases in common iliac LN, two in the internal iliac LN area, three in the external LN area, seven in the inguinal LN area, six in the presacral area and two in the mesorectal space. In 5/37 (14\%) cases there was complete accordance between 18FDG-PET/CT and MRI in detecting LN involvement (Table 2). A mapping of number of patients with involved LN was carried out, mainly comparing MRI and 18FDG-PET/CT results (Figure 1).

TABLE 1 | Patient and tumor characteristics.

\begin{tabular}{lc}
\hline Total number & $\mathbf{3 7}$ \\
\hline Gender & $28 \mathrm{~F}$ \\
Median age & 95 (range 40-88) \\
Performance status & 30 ECOGO \\
Histology & 7 ECOG1 \\
Clinical stage (NCCN 2020) & 37 SCC \\
& 1 T1NOMO I \\
& 1 T1N1MO IIIA \\
& 6 T2NOMO IIA \\
& 6 T2N1MO IIIA \\
& 1 T3NOMO IIB \\
& 10 T3N1MO IIIC \\
10 T4N1MO IIIC \\
1 T2N1M1 IV \\
1 T3N1M1 IV
\end{tabular}

The 18FDG-PET/CT planning led to change the stage in five (14\%) cases when compared to MRI, particularly in four (11\%) cases the stage changed from N0 to N1 for positive inguinal LN and in one $(2.5 \%)$ case from M0 to M1 for common iliac LN involvement. In $10 / 20(50 \%)$ patients with positive mesorectal LN, MRI outperformed 18FDG-PET/CT in detecting LN in this area. The 18FDG-PET/CT helped us to target volume delineation: in one case with PET-positive common iliac LN, the CTV was extended cranially to include this area (Figure 2). In 14/37 (38\%) patients 18FDG-PET/CT led to a dose escalation on PET-positive $\mathrm{LN}$ reaching 50-54 Gy.

\section{DISCUSSION}

In clinical staging of anal cancer, MRI represents the gold standard to detect the tumor lesion, the involved adjacent anatomical structures and the adjacent loco-regional lymphatic spread. The abdominal and pelvic lymphadenopathies and distant metastasis are usually assessed with contrast enhanced CT. In the recent years the role of 18FDG-PET/CT in the staging of SCCAC has been growing probably due to high FDG-avidity of SCCAC as reported in the literature.

According to the NCCN guidelines, 18FDG-PET/CT may be considered to verify anal canal cancer staging especially to evaluate pelvic LN with normal size on CT imaging. Similarly, in the ESMO guidelines 18FDG-PET/CT is considered optional but is often recommended (1).

A recent meta-analysis of 17 studies compared the role of PET/ CT with conventional imaging in the staging, response evaluation and follow up of patients with anal canal cancer. The authors calculated the pooled sensitivity and specificity for detection of LN involvement by 18 FDG-PET/CT at 93 and 76\%, respectively (7). Moreover, several studies have shown that 18 FDG-PET/CT led to upstaging in about $20 \%$ of cases changing TNM stage in $21 \%$ and altering treatment strategy in 3-5\% of cases $(1,12-16)$.

The use of 18FDG-PET/CT may also impact on radiotherapy planning as shown in three systematic reviews and meta-analyses where treatment planning was modified from 12 to $59 \%$ of patients based on PET/CT results (7, 17-19).

The present study, similarly to Krengli et al., aims to analyze the potential impact of 18FDG-PET/CT on tumor staging and treatment strategy in the management of SCCAC and investigates how 18FDG-PET/CT changed volume delineation in the radiotherapy treatment planning (20).

According to the literature, we found that MRI resulted more sensitive in T staging and provided more details to local extension, remaining the modality of choice for primary GTV contouring (21).

TABLE 2 | Distribution of positive lymph-nodes in our sample of patients detected with 18FDG-PET/CT and/or MRI.

IMAGING

LYMPH NODES (number of cases)

\begin{tabular}{cccccccc} 
& INGUINAL & COMMON ILIAC & INTERNAL ILIAC & OBTURATOR & EXTERNAL ILIAC & PRESACRAL & MESORECTAL \\
\hline MRI- PET+ & 6 & 3 & 2 & 0 & 3 & 6 \\
MRI+PET+ & 13 & 2 & 0 & 0 & 1 & 2 \\
MRI+ PET- & 3 & 1 & 4 & 6 & 3 & 3 & 2 \\
\end{tabular}




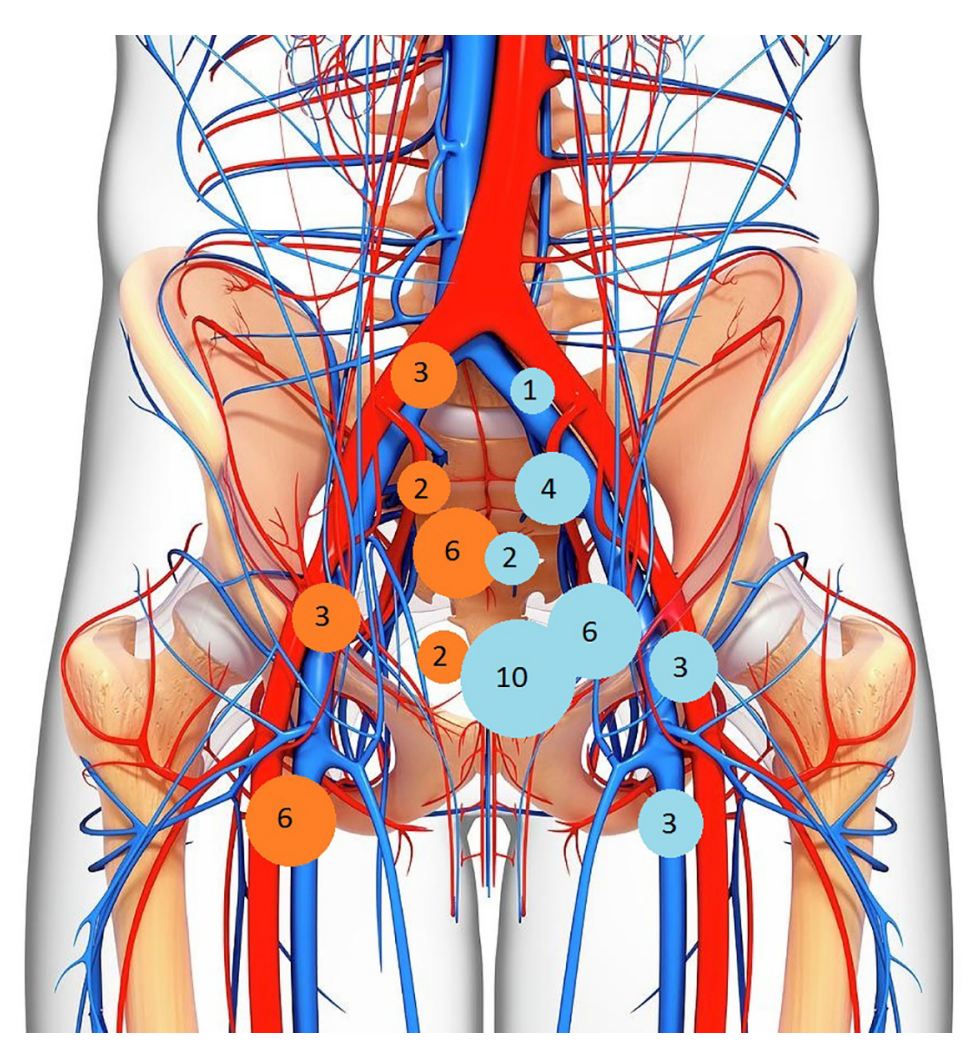

FIGURE 1 | Mapping of cases with PET-positive (orange) and MRI-positive (light-blue) lymph nodes.

On the other hand, in our study we observed that 18FDGPET/CT had a higher implication on nodal staging, particularly on regard to inguinal $\mathrm{LN}$, leading to upstaging in the $14 \%$ of cases: in four (11\%) from N0 to N1 for positive inguinal LN and in one (3\%) from M0 to M1 for common iliac LN involvement.

Similar results were obtained in a study by Zimmerman et al. that evaluated 26 patients and reported about $13 \%$ of upstaging. Analogous results are showed in a recent meta-analysis by Jones and colleagues, with upstaging rate of $15 \%$. Mahmud systematically review the literature to investigate the utility of 18FDG-PET/CT in the clinical staging and found that PET/CT identified distant metastatic sites not seen on conventional imaging in 2.4 to $4.7 \%$ of cases in agreement with our data $(2.5 \%)$. No case of downstaging was reported in our series contrary to the literature where the use of 18FDG-PET/CT led to about $15 \%$ of downstaging $(7,21)$.

The influence of 18FDG-PET/CT findings on target volume definition and treatment planning is quite variable. Bhuva et a reported a series of 43 patients undergone 18FDG-PET/CT in addition to routine CT and MRI (8). The 18FDG-PET/CT imaging altered nodal stage in $32 \%$ of cases; however, despite these findings, all treatment plannings were not modified. In the study by de Winton and colleagues, 18FDG-PET/CT changed the management in $16 \%(10 / 61)$ of cases. Particularly, the addition of $18 \mathrm{FDG}-\mathrm{PET} / \mathrm{CT}$ to clinical staging had a considerable impact on treatment intent in $3 \%$ of patients and changed radiotherapy fields or technique, including or not nodal disease, in $13 \%$ of cases (18). Nguyen et al. analyzed 50 patients with SCCAC where pre-treatment 18FDG-PET/CT identified additional involved nodal groups causing radiotherapy treatment planning amendments in 19\% (19).

In our sample, 18FDG-PET/CT allowed a dose escalation on PET positive LN in 14 (38\%) patients using SIB.

Krengli et al. reported that 18FDG-PET/CT changed GTV and CTV contours in 55 and $37 \%$ of cases, respectively, with high rate of local control and low rate of late toxicity (20). Drapper et al. carried out a retrospective study of thirty-seven patients and compared three different contouring guidelines for pelvic LN. They showed how 18FDG-PET/CT imaging changed the contouring of LN areas and that LN "misses" generally appeared cranially (common iliac or para-aortic) or caudally (inguinal) to the recommended CTVs (22).

A recent study by Fiorentino et al., analyzed the role of 18FDG-PET/CT for the radiotherapy planning definition of the biological target volume in several pathologies including anal cancer. They considered 18FDG-PET/CT for anal cancer a useful supplement in target definition for delineating smaller volume compared to CT alone and similar GTVs in comparison of MRI (23).

In our study, similarly to Drapped et al., 18FDG-PET/CT led to modify target volume of CTV N, particularly inguinal, external iliac and common iliac LN contours to include all PET-positive LN (9-11). No changes have been made to GTV $\mathrm{T}$ and CTV $\mathrm{T}$ delineation. 

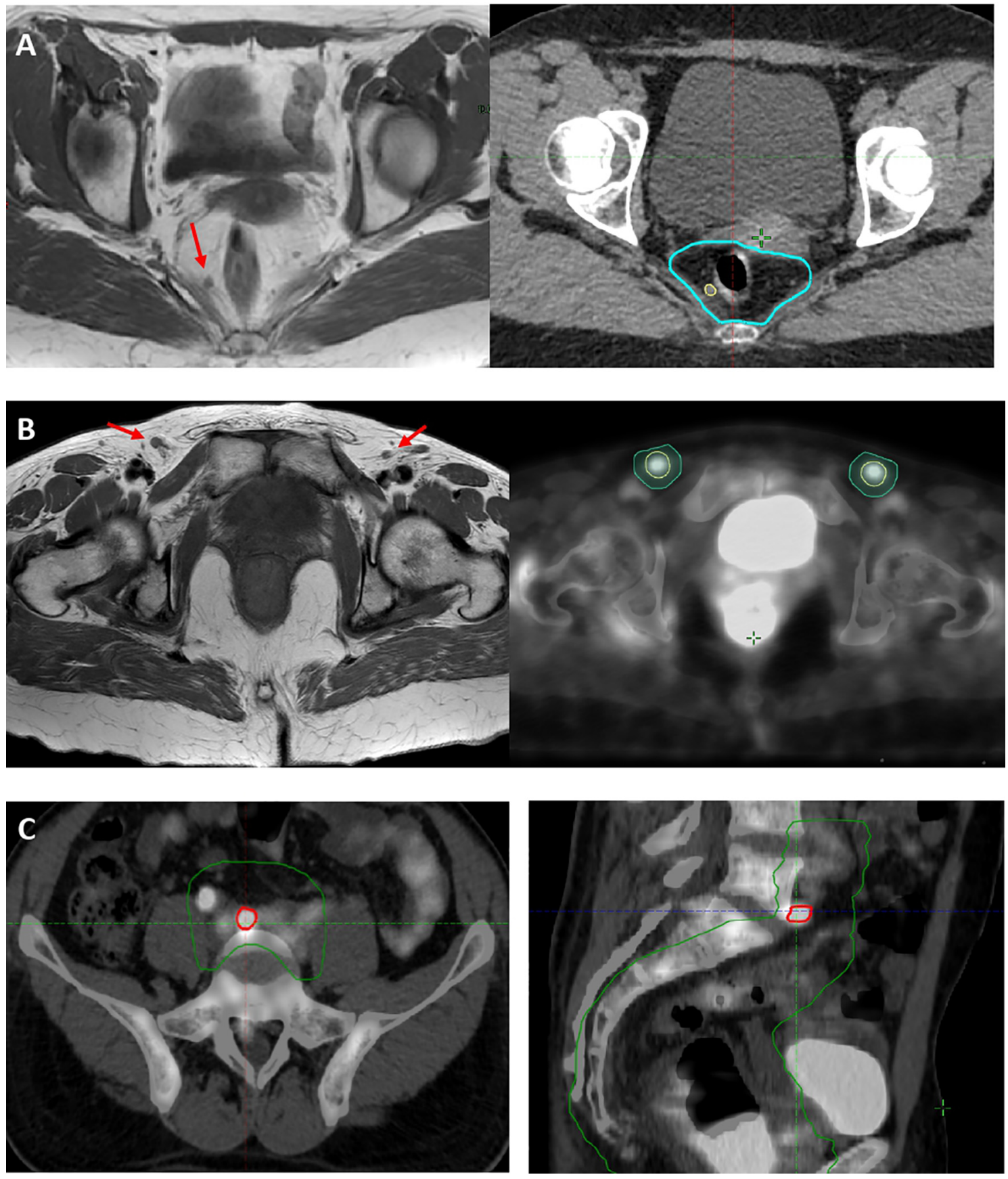

FIGURE 2 | (A) Case of mesorectal PET-/MRI+ LN (red arrow), included as mesorectal GTV N (yellow line) in 54 Gy high-dose volume (light blue line). (B) Case of disease upstage and dose escalation on MRI- (red arrows)/PET+ (yellow line) inguinal LN. (C) Case of disease upstage with a dose escalation on MRI-/PET+ common iliac LN (red line) and inclusion of common iliac LN level to 45 Gy low-dose volume (green line).

\section{CONCLUSION}

In conclusions, 18FDG-PET/CT plays an important role in the detection of LN in patients affected by anal cancer. This could lead to a precise definition of radiotherapy target volume and dose-escalation improving tumor control. The results of our studies are in accordance with other series reported in Literature showing the usefulness of 18FDG-PET/CT in the initial staging of patients. More investigations are needed to define the role of 18FDG-PET/CT in the target volume delineation of anal cancer. 


\section{DATA AVAILABILITY STATEMENT}

The raw data supporting the conclusions of this article will be made available by the authors, without undue reservation.

\section{ETHICS STATEMENT}

Ethical review and approval was not required for the study on human participants in accordance with the local legislation and institutional requirements. The patients/participants [legal guardian/next of kin] provided written informed consent to participate in this study.

\section{REFERENCES}

1. Glynne-Jones R, Nilsson PJ, Aschele C, Goh V, Peiffert D, Cervantes A, et al. European Society for Medical Oncology (ESMO); European Society of Surgical Oncology (ESSO); European Society of Radiotherapy and Oncology (ESTRO). Anal Cancer: ESMO-ESSO-ESTRO Clinical Practice Guidelines for Diagnosis, Treatment and Follow-Up. Eur J Surg Oncol (2014) 40(10):1165-76. doi: 10.1016/j.ejso.2014.07.030

2. Benson AB, Venook AP, Al-Hawary MM, Cederquist L, Chen YJ, Ciombor KK, et al. Anal Carcinoma, Version 2.2018, NCCN Clinical Practice Guidelines in Oncology. J Natl Compr Canc Netw (2018) 16(7):852-71. doi: 10.6004/jnccn.2018.0060

3. Symer MM, Yeo HL. Recent Advances in the Management of Anal Cancer. F1000Res (2018) 28:7. doi: 10.12688/f1000research.14518.1. F1000 Faculty Rev-1572.

4. Kochhar R, Plumb AA, Carrington BM, Saunders M. Imaging of Anal Carcinoma. AJR Am J Roentgenol (2012) 199(3):W335-44. doi: 10.2214/AJR.11.8027

5. Caldarella C, Annunziata S, Treglia G, Sadeghi R, Ayati N, Giovanella L. Diagnostic Performance of Positron Emission Tomography/Computed Tomography Using Fluorine-18 Fluorodeoxyglucose in Detecting Locoregional Nodal Involvement in Patients With Anal Canal Cancer: A Systematic Review and Meta-Analysis. Sci World J (2014) 2014:196068. doi: 10.1155/2014/196068

6. Mistrangelo M, Pelosi E, Bellò M, Ricardi U, Milanesi E, Cassoni P, et al. Role of Positron Emission Tomography-Computed Tomography in the Management of Anal Cancer. Int J Radiat Oncol Biol Phys (2012) 84(1):6672. doi: 10.1016/j.ijrobp.2011.10.048

7. Mahmud A, Poon R, Jonker D. PET Imaging in Anal Canal Cancer: A Systematic Review and Meta-Analysis. Br J Radiol (2018) 90(1080):20170370. doi: 10.1259/bjr.20170370

8. Bhuva NJ, Glynne-Jones R, Sonoda L, Wong WL, Harrison MK. To PET or Not to PET? That Is the Question. Staging in Anal Cancer. Ann Oncol (2012) 23(8):2078-82. doi: 10.1093/annonc/mdr599

9. Ng M, Leong T, Chander S, Chu J, Kneebone A, Carroll S, et al. Australasian Gastrointestinal Trials Group (AGITG) Contouring Atlas and Planning Guidelines for Intensity-Modulated Radiotherapy in Anal Cancer. Int J Radiat Oncol Biol Phys (2012) 83(5):1455-62. doi: 10.1016/j.ijrobp.2011.12.058

10. Myerson RJ, Garofalo MC, El Naqa I, Abrams RA, Apte A, Bosch WR, et al. Elective Clinical Target Volumes for Conformal Therapy in Anorectal Cancer: A Radiation Therapy Oncology Group Consensus Panel Contouring Atlas. Int J Radiat Oncol Biol Phys (2009) 74(3):824-30. doi: 10.1016/j.ijrobp. 2008.08.070

11. Valentini V, Gambacorta MA, Barbaro B, Chiloiro G, Coco C, Das P, et al. International Consensus Guidelines on Clinical Target Volume Delineation in Rectal Cancer. Radiother Oncol (2016) 120(2):195-201. doi: 10.1016/ j.radonc.2016.07.017

12. Chulroek T, Kordbacheh H, Wangcharoenrung D, Cattapan K, Heidari P, Harisinghani MG. Comparative Accuracy of Qualitative and Quantitative 18F-

\section{AUTHOR CONTRIBUTIONS}

GM, CC, and MB developed the study design, collected, and interpreted patients' data. $\mathrm{AP}$ and $\mathrm{LB}$ provided and interpreted the PET-imaging data. CC, GM, SC, and LV drafted the manuscript. $\mathrm{CC}, \mathrm{LV}, \mathrm{MB}, \mathrm{SC}, \mathrm{AP}$, and GM contributed to the interpretation of the results and discussion. All authors contributed to the article and approved the submitted version.

\section{FUNDING}

This work was supported by Azienda Ospedaliera Ospedali Riuniti Di Ancona.

FDG PET/CT Analysis in Detection of Lymph Node Metastasis From Anal Cancer Abdom Radiol (NY) (2019) 44(3):828-35. doi: 10.1007/s00261-019-01907-6

13. Cotter SE, Grigsby PW, Siegel BA, Dehdashti F, Malyapa RS, Fleshman JW, et al. FDG-PET/CT in the Evaluation of Anal Carcinoma. Int J Radiat Oncol Biol Phys (2006) 65(3):720-5. doi: 10.1016/j.ijrobp.2006.01.009

14. Pepek JM, Willett CG, Czito BG. Radiation Therapy Advances for Treatment of Anal Cancer. J Natl Compr Canc Netw (2010) 8(1):123-9. doi: 10.6004/ jnccn.2010.0008

15. Trautmann TG, Zuger JH. Positron Emission Tomography for Pretreatment Staging and Posttreatment Evaluation in Cancer of the Anal Canal. Mol Imaging Biol (2005) 7(4):309-13. doi: 10.1007/s11307-005-0003-6

16. Jones M, Hruby G, Solomon M, Rutherford N, Martin J. The Role of FDGPET in the Initial Staging and Response Assessment of Anal Cancer: A Systematic Review and Meta-Analysis. Ann Surg Oncol (2015) 22(11):357481. doi: 10.1245/s10434-015-4391-9

17. Albertsson P, Alverbratt C, Liljegren A, Björkander E, Strandell A, Samuelsson O, et al. Positron Emission Tomography and Computed Tomographic (PET/ CT) Imaging for Radiation Therapy Planning in Anal Cancer: A Systematic Review and Meta-Analysis. Crit Rev Oncol Hematol (2018) 126:6-12. doi: 10.1016/j.critrevonc.2018.03.013

18. Winton E, Heriot AG, Ng M, Hicks RJ, Hogg A, Milner A, et al. The Impact of 18-Fluorodeoxyglucose Positron Emission Tomography on the Staging, Management and Outcome of Anal Cancer. Br J Cancer (2009) 100(5):693700. doi: 10.1038/sj.bjc.6604897

19. Nguyen BT, Joon DL, Khoo V, Quong G, Chao M, Wada M, et al. Assessing the Impact of FDG-PET in the Management of Anal Cancer. Radiother Oncol (2008) 87(3):376-82. doi: 10.1016/j.radonc.2008.04.003

20. Krengli M, Milia ME, Turri L, Mones E, Bassi MC, Cannillo B, et al. FDGPET/CT Imaging for Staging and Target Volume Delineation in Conformal Radiotherapy of Anal Carcinoma. Radiat Oncol (2010) 5:10. doi: 10.1186/ 1748-717X-5-10

21. Zimmermann M, Beer J, Bodis S, von Moos R, Vlachopoulou V, Zwahlen DR, et al. PET-CT Guided SIB-IMRT Combined With Concurrent 5-FU/MMC for the Treatment of Anal Cancer. Acta Oncol (2017) 56(12):1734-40. doi: 10.1080/0284186X.2017.1325003

22. Dapper H, Schiller K, Münch S, Peeken JC, Borm K, Weber W, et al. Have We Achieved Adequate Recommendations for Target Volume Definitions in Anal Cancer? A PET Imaging Based Patterns of Failure Analysis in the Context of Established Contouring Guidelines. BMC Cancer (2019) 19(1):742. doi: 10.1186/s12885-019-5970-0

23. Fiorentino A, Laudicella R, Ciurlia E, Annunziata S, Lancellotta V, Mapelli P, et al. AIRO Giovani - Italian Association of Radiation Oncology-Young Members and AIMN -Italian Association of Nuclear Medicine- Young Members Working Group. Positron Emission Tomography With Computed Tomography Imaging (PET/CT) for the Radiotherapy Planning Definition of the Biological Target Volume: PART 2. Crit Rev Oncol Hematol (2019) 139:117-24. doi: 10.1016/j.critrevonc.2019.03.008 
Conflict of Interest: The authors declare that the research was conducted in the absence of any commercial or financial relationships that could be construed as a potential conflict of interest.

Copyright (C) 2021 Di Carlo, di Benedetto, Vicenzi, Costantini, Cucciarelli, Fenu, Arena, Mariucci, Montisci, Panni, Patani, Valenti, Palucci, Burroni and Mantello.
This is an open-access article distributed under the terms of the Creative Commons Attribution License (CC BY). The use, distribution or reproduction in other forums is permitted, provided the original author(s) and the copyright owner(s) are credited and that the original publication in this journal is cited, in accordance with accepted academic practice. No use, distribution or reproduction is permitted which does not comply with these terms. 